\title{
Cell Membrane-Based Nanoreactor To Mimic the Bio- Compartmentalization Strategy of a Cell
}

Vimalkumar Balasubramanian, ${ }^{\dagger, \ddagger}$ Andrea Poillucci, ${ }^{\dagger, \ddagger}, \S$ Alexandra Correia, ${ }^{\dagger}$ Hongbo Zhang, ${ }^{\perp, \|}$ Christian Celia, ${ }^{*},, \#$ and Hélder A. Santos ${ }^{*}, \triangle^{\prime},(1)$

${ }^{*}$ Drug Research Program, Division of Pharmaceutical Chemistry and Technology, Faculty of Pharmacy, and $\triangle_{\text {Helsinki Institute of }}$ Life Science (HiLIFE), University of Helsinki, Viikinkaari 5E, Helsinki FI-00014, Finland

${ }^{\S}$ Department of Pharmacy, University of Chieti-Pescara “G. d'Annunzio”, Via dei Vestini 31, Chieti I-66100, Italy

${ }^{\perp}$ Department of Pharmaceutical Science, Åbo Akademy University, BioCity, Artillerigatan 6A, Turku FI-20520, Finland

"Turku Center of Biotechnology, Åbo Akademi University, Tykistokatu 6, Turku FI-20520, Finland

\#Department of Nanomedicine, Houston Methodist Research Institute, 6670 Bertner Avenue, Houston, Texas 77030, United States

\section{Supporting Information}

ABSTRACT: Organelles of eukaryotic cells are structures made up of membranes, which carry out a majority of functions necessary for the surviving of the cell itself. Organelles also differentiate the prokaryotic and eukaryotic cells, and are arranged to form different compartments guaranteeing the activities for which eukaryotic cells are programmed. Cell membranes, containing organelles, are isolated from cancer cells and erythrocytes and used to form biocompatible and longcirculating ghost nanoparticles delivering payloads or catalyzing enzymatic reactions as nanoreactors. In this attempt, red blood cell membranes were isolated from erythrocytes, and engineered to form nanoerythrosomes (NERs) of $150 \mathrm{~nm}$. The horseradish peroxidase, used as an enzyme model, was loaded inside the aqueous compartment of NERs, and its catalytic reaction with Resorufin was monitored. The resulting nanoreactor protected the enzyme from proteolytic degradation, and potentiated the enzymatic reaction in situ as demonstrated by maximal velocity $\left(V_{\max }\right)$ and Michaelis constant $\left(K_{\mathrm{m}}\right)$, thus suggesting the high catalytic activity of nanoreactors compared to the pure enzymes.

KEYWORDS: nanoparticle reactors, nanoerythrosomes, colloidal nanoparticles, enzyme reaction, self-assembled membrane vesicles

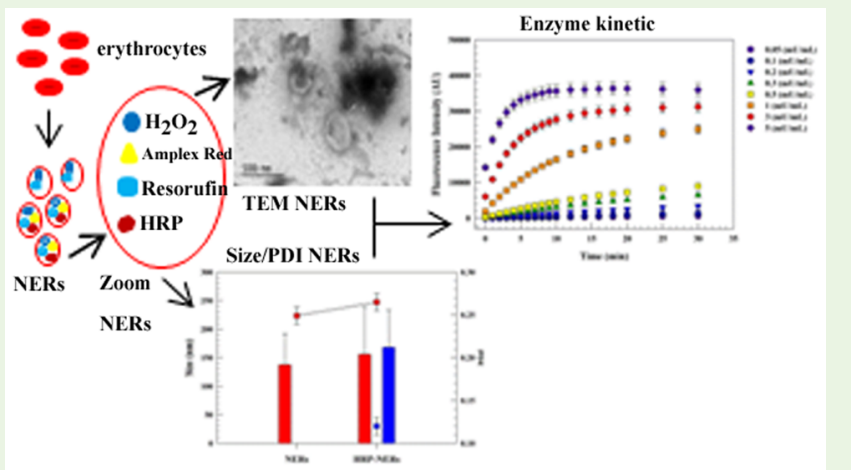

\section{INTRODUCTION}

Synthetic biology offers plenty of exciting and potential possibilities to have deeper insights, and to understand the fundamentals of "origin of life", and various biological processes required for the cell functions. ${ }^{1-5}$ Inspired by living cells, scientists intend to create synthetic cellular structures to fundamentally understand the complex biological process involved in the maintenance of life. ${ }^{5}$ These synthetic cellular systems are termed as protocells or artificial organelles/cells. ${ }^{1-3}$ Protocells are biomimetic structures that aim to mimic the properties of biological cells in order to study cellular functions or to be used as bioreactors for biotechnological or drug delivery applications. These protocells consist of an aqueous compartment that can enclose the machinery for a certain biological process delimited by a membrane that should preferably be semipermeable or selective-permeable, to allow the communication with the external environment. Protocells are beneficial for isolating the biological process from undesired external factors, as well as to keep the elements of the biological process close to each other and to regulate the transport of molecules in and out of the cellular environment. ${ }^{1}$ Particularly, biocompartmentalization is the essential component of the cells, in which different proteins and enzymes can easily transport molecules and signals to perform various metabolic (biochemical) reactions required for the cell functions. ${ }^{1-7}$ In the interest of mimicking nature's compartmentalization strategies, cell membranes, forming vesicle structures, have gained tremendous attention because of the reaction space available in the interior of aqueous compartment and at the interface between membrane and the exterior. ${ }^{1}$ At the same time, vesicle membranes can enclose proteins and enzymes for a certain biochemical reaction and allow the communication with the exterior. In the present study, we aim to develop cell membrane based nanoreactors loaded with enzymes that will allow enzymatic reactions in the aqueous compartment without the requirement of releasing the enzymes to perform the

Received: December 4, 2017

Accepted: February 15, 2018

Published: February 15, 2018 
reactions. In this way, the design of cell membrane-based nanoreactors can protect the sensitive enzymes from proteolytic degradation, and simultaneously allow the catalytic reactions inside the compartment or the transport of desired compounds from the reaction end-product to the outside of the nanoreactors. ${ }^{1}$ The concept of cell membrane based nanoreactors will mimic the compartmentalization strategies of a cell.

To attain this, our approach was to use cell membrane derived materials through biomimetic engineering to mimic nature biocompartmentalization strategy. In particular, the selected source materials were breast cancer cells (MDA-MB231) and red blood cells (RBCs), in order to take advantage of the homotypic binding of cancer cells, to achieve an innovative strategy of active targeting, ${ }^{8,9}$ and the natural biocompatibility and long systemic circulation of RBCs. ${ }^{10,11}$ Moreover, the RBCs have also been selected for the possibility to be further engineered from ghost erythrocytes membranes to form nanoerythrosomes (NERs), i.e., derivatives of RBCs with an average diameter of $100 \mathrm{~nm} .^{12}$ In fact, the most interesting feature of NERs is their capability to avoid the reticuloendothelial clearance and thus making "stealth" nanocarriers. ${ }^{10,11}$ The physicochemical properties of the cell membrane derived vesicles have been investigated in order to study their possible use as a biomimetic drug delivery system, and especially as a miniaturized reaction vessel, i.e., as a "nanoreactor". ${ }^{13}$ To date, RBCs have already been used as a circulating bioreactor for the clearance of undesired substance from the organism, ${ }^{14,15}$ as enzyme transporters for enzyme replacement therapy, ${ }^{15}$ whereas cancer cell membrane derived material has also been investigated as artificial organelle. ${ }^{13}$ Nevertheless, pure cancer cell membrane, without the integration and support of polymersomes, ${ }^{1}$ and mesoporous silicon nanoparticles, ${ }^{13}$ has not been investigated yet for these purposes.

Therefore, cancer and red blood cell membranes have been derived from their source material and used to form vesicles, and their physicochemical properties have been studied. The ultimate goal of this study was to investigate if both the cell membrane derived materials were suitable to be used as a biomimetic drug delivery system and as a nanoreactor. It is important to highlight that, even though it is definitely possible to form nanosized vesicles with a narrow size distribution from both cancer cells and red blood cells, actually only NERs can be used to efficiently entrap a payload for therapeutic applications. Previous studies already signalize that using the same cancer cell alone, without the support of inorganic nanoparticles, could not act as an autonomous system for the loading of a therapeutic cargo, because of resulted insufficient entrapment unstable. ${ }^{13}$ Although the vesicles were formed in both cases by natural lipid derivatives, the best performances were possible using erythrocyte membrane because of their unique structure. ${ }^{16}$ In fact, NERs membrane has a thickness of $\sim 11$ $\mathrm{nm},{ }^{17}$ and is more suitable for drug delivery applications than the breast cancer cell membranes $(\sim 4 \mathrm{~nm}),{ }^{13}$ which is too thin and thus useful only for the coating of nanoparticles. ${ }^{8,13}$

NERs have thus been used as a host material to entrap an enzyme model, the Horseradish Peroxidase (HRP), which provides the microenvironment for the confinement of enzyme molecules, in order to mimic nature compartmentalization strategies. As previously reported by other scientists, the enzyme compartmentalization offered the double benefit of protecting the enzyme and allowing to act "in situ", without the requirement of releasing it. ${ }^{18}$ Finally, to study the catalytic efficiency of the nanoreactor, enzyme kinetic constants $\left(V_{\max }\right.$ and $K_{\mathrm{m}}$ ) have been calculated to compare the reaction rates between an HRP-catalyzed reaction inside the nanoreactor with the same reaction studied in a solution of the pure enzyme. These experiments were performed to understand how the biocompartmentalization process could affect the rate of the enzymatic reaction.

\section{MATHERIALS AND METHODS}

Details of all experimental procedures and characterizations of the resulting breast cancer cells derived vesicles and NERs were included in the Supporting Information.

\section{RESULTS AND DISCUSSION}

Physicochemical Characterization of Nanocarriers. The physicochemical characterization of the vesicular nanocarriers is probably the most important parameter to study because it is strictly related to their final properties and way of administration. In fact, size, shape, size distribution and zeta potential of colloidal nanoparticles can affect their fate after systemic injection, and modulate distributions through passive targeting. ${ }^{19-22}$ Furthermore, surface properties, ${ }^{23-25}$ and modifications, ${ }^{26-28}$ of colloidal nanoparticles can affect their potentialities in therapy, control pharmacokinetic and biodistribution, and target selectively cells and tissues.

Nanoerythrosomes, as already reported in literature, have great potential in nanomedicine as circulating bioreactors, ${ }^{14,15}$ artificial organelles, ${ }^{13}$ drug carrier for parasitic diseases, ${ }^{12,29}$ and for enzyme replacement therapy; ${ }^{15}$ but the most important application is indeed for cancer therapy. Although the pure ghost erythrocytes have been used successfully to treat or detect solid tumors, their micrometer size $(\approx 7-8 \mu \mathrm{m})$ limits the extravascular diffusion of these carriers, as well as their chance to directly approach tumor cells, thus making active tumor cell-targeted drug delivery almost impossible. ${ }^{12}$ In fact, nanoparticle based drug delivery systems must be thus smaller than $200 \mathrm{~nm}$, as reported in literature; ${ }^{30}$ that is why the size and PDI are crucial to be investigated. PolyDispersity Index (PDI) is a parameter, which indicates the size distribution of nanoparticles. PDI below 0.3 shows homogeneous nanoparticle size distribution, whereas nanoparticles with a PDI over 0.3 are broad size distributed and contain large particles or aggregates that can slowly form a bottom sediment. ${ }^{30}$

The average sizes and PDI of cancer cells derived vesicles and NERs were optimized combining different procedures as previously reported. ${ }^{31}$ In particular, they were synthesized using thin layer evaporation (TLE) method combined with extrusion and/or sonication, or directly by extrusion through a polycarbonate membrane filter pore by $8-10$ consecutive extrusions under nitrogen pressure in a thermostatically controlled extrusion device at a $37{ }^{\circ} \mathrm{C}$. These procedures allow us to get nanoparticles with average sizes and PDI depending on the lipid compositions and preparation procedures. $^{31}$ The combination of TLE with extrusion and/or sonication affects the physicochemical parameters of both the colloidal nanoparticles.

The cancer cell derived vesicles made up by TLE had an average size of $135 \pm 62 \mathrm{~nm}$ and a PDI of $0.195 \pm 0.01$ (Table $1)$. Even though size and PDI measurements made them suitable for drug delivery applications, they were excluded as potential candidates for the loading of the enzyme, because the membranes were thin and thus useful only for the coating of nanoparticles as herein reported. ${ }^{8,13}$ In fact, we previously 
Table 1. Physicochemical Characterization of Breast Cancer Cell (MDA-MB-231) Membrane-Based Vesicles and Nanoerythrosomes (NERs) Derived from Red Blood Cells $(\mathrm{RBCs})^{a}$

\begin{tabular}{|c|c|c|c|}
\hline formulations & sizes $(\mathrm{nm})$ & $\mathrm{PDI}^{b}$ & $\mathrm{ZP}^{c}(\mathrm{mV})$ \\
\hline $\begin{array}{l}\mathrm{TLE}^{d}-\mathrm{MDA}-\mathrm{MB}-231 \text { cell } \\
\text { membrane vesicles }\end{array}$ & $135 \pm 62$ & $0.195 \pm 0.01$ & $-21 \pm 4$ \\
\hline $\mathrm{TLE}^{d}-\mathrm{NERs}^{e}$ & $478 \pm 47$ & $0.669 \pm 0.03$ & $-34 \pm 1$ \\
\hline $\begin{array}{l}\text { sonication/extrusion MDA-MB- } \\
231 \text { vesicles }\end{array}$ & $85 \pm 79$ & $0.404 \pm 0.02$ & $-21 \pm 3$ \\
\hline sonication/Extrusion NERs ${ }^{e}$ & $138 \pm 54$ & $0.249 \pm 0.01$ & $-30 \pm 4$ \\
\hline $\begin{array}{l}\mathrm{HPR}^{f}-\mathrm{NERs}^{e} \text { (before } \\
\text { purification) }\end{array}$ & $156 \pm 82$ & $0.265 \pm 0.01$ & $-33 \pm 1$ \\
\hline $\begin{array}{l}\mathrm{HPR}^{f}-\mathrm{NERs}^{e} \text { (after } \\
\text { purification) }\end{array}$ & $168 \pm 66$ & $0.120 \pm 0.01$ & $-31 \pm 2$ \\
\hline
\end{tabular}

${ }^{a}$ Average sizes, narrow size distribution (Polydispersity Index), and zeta potential of colloidal nanoparticles were measured by dynamic light scattering analysis using a Zetasizer Nano ZS (Malvern, UK). Results are the mean \pm standard deviation from at least three independent measurements. ${ }^{b}$ Polydispersity index (PDI). ${ }^{c}$ Zeta potential (ZP). ${ }^{d}$ Thin layer evaporation (TLE). ${ }^{e}$ Nanoerythrosomes (NERs). ${ }^{f}$ Horseradish peroxidase (HRP).

demonstrated that vesicles originated from cancer cell membranes without the pain support of undecylenic acid modified thermally hydrocarbonized PSi (UnPSi) nanoparticles cannot form nanovesicles with a bilayer structure capable to effective loading enzymes. ${ }^{13}$ This evidence demonstrated that UnPSi, as well as other nanoparticles, are crucial components in the design of nanoreactors supporting the enzymatic activity and catalysis. UnPSi also support the partition of enzymes in the nanoreactors mimicking artificial organelle structure, and improve the performances of enzyme kinetic that was not allowed with nanoreactors of thin layer cancer cell membrane vesicles. ${ }^{13}$ The stabilization of cancer cell membrane vescicles with UnPSi also maintains the intactness and stability of the structure. $^{13}$ Similar results were obtained constructing hierarchical yolk-shell@shell structured bifunctional mesoporous silica nanoreactor with $\mathrm{Pd}$ nanoparticles deposited in the inner core and enzymes adsorbed in the outer shell. ${ }^{32}$ The architecture of hybrid shell-core nanoconstruct increases the enzymatic catalysis of enzymes (amine) inside the nanoreactors due to the a complete spatial separation of metal active components and substrates and the right orientation of the desired diffusion pathway of the reaction sequence. ${ }^{32}$

The average thickness of the bilayer forming stable nanovesicles is $5.58 \mathrm{~nm}$ and the variations in the thickness are within $0.6 \mathrm{~nm}$. This minimum value is higher than $\sim 4 \mathrm{~nm},{ }^{13}$ obtained using cancer cell associated membranes, and corresponded to molecules in a highly defective state that bridges the interdigitated region of the bilayer on one leaflet with the subsequent noninterdigitated region. This thickness indicated the development of complicated correlations between height, headgroup density, tilt, and tail conformation in order to accommodate the rippling in the bilayer, and generated nanovesicles unsuitable for delivering active agents. ${ }^{33}$ Additionally, theoretical experiments demonstrated that asymmetric bilayers separated by $6 \mathrm{~nm}$ required less energy to allow the fusion pore formation, and can promote fusion of membranes by a large scale deformations of lipid bilayers and a relative increase in kinetic reaction. ${ }^{34}$

Cancer-cell-derived vesicles obtained using only the extrusion procedure showed an average size of $85 \pm 79 \mathrm{~nm}$, and a PDI of $0.404 \pm 0.02$ (Table 1). In this specific case, the size of resulting vesicles was quite small and thus these nanoparticles could be used as a biomimetic drug delivery system; unfortunately, their PDI value was beyond the limit of 0.3 for narrow size distributed particles. It means that the cancer-cellderived vesicle population was polydisperse, ${ }^{30}$ as also confirmed by transmission electron microscopy (TEM) images (Figure 1A, B). TEM images proved the limited thickness of the cancer

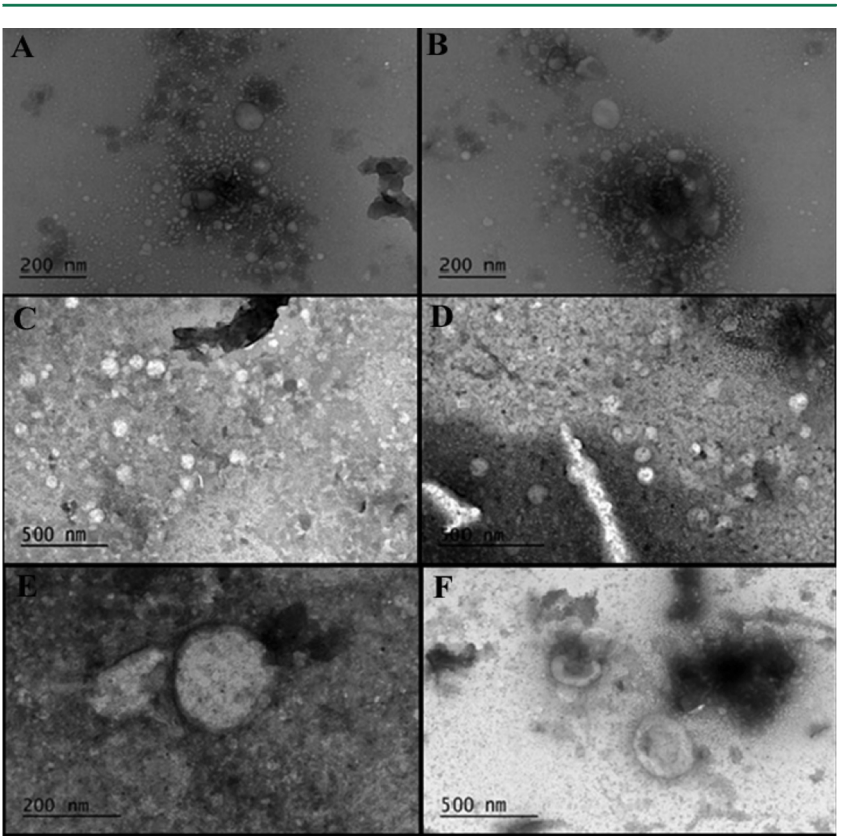

Figure 1. Transmission electron microscopy (TEM) photos of (A, B) cancer cell derived vesicles and $(\mathrm{C}-\mathrm{F})$ empty NERs, obtained using only the sonication/extrusion techniques. The thickness and polydispersity of the cancer cell derived vesicles $(\approx 4 \mathrm{~nm})$ and NERs $(\approx 11 \mathrm{~nm})$ were analyzed using the TEM images and further compared. Vesicles obtained from (A, B) cancer cell membrane derivatives are broad size distributed, whereas (C, D) NERs are size distributed. (E) The zoom of NERs allows appreciating the narrow size distribution and the spherical shape of nanovesicles. (F) NERs preserved the characteristic discoidal biconcave shape of RBCs. The TEM photos are representative of three different and independent analyses.

cell derived vesicles $(\approx 4 \mathrm{~nm}),{ }^{13}$ formed by direct extrusion, compared to NERs, and also their broad size distribution (Figures 1A, B). Furthermore, TEM images showed that empty NERs (Figure $1 \mathrm{C}-\mathrm{F}$ ) were well distributed along the grid, presented a narrow size distribution, and a thick membrane $(\approx$ $11 \mathrm{~nm}),{ }^{17}$ suitable for drug delivery applications (Figure 1CF). Interestingly, NERs also preserved the characteristic biconcave discoidal shape of RBCs (Figure 1F).

For this reason, cancer-cell-derived nanocarriers have been excluded for the further experiments, and only NERs were used to test enzyme kinetics.

A completely opposite situation could be observed for the NERs, made up using TLE method, which had an average size of $478 \pm 47 \mathrm{~nm}$ and a PDI of $0.669 \pm 0.03$ (Table 1 ). This size was bigger than $200 \mathrm{~nm}$ and it might not be suitable to achieve long-circulating nanoparticles for systemic injection. Furthermore, the PDI was more than two times bigger than the limit of 0.3 , which provided stable and narrow size distributed colloidal nanoparticles. ${ }^{30}$ On the basis of these physicochemical properties, NERs, made up from TLE method, were excluded as potential candidates for further experiments. 
Instead, NERs made up only by extrusion, showed an average particle size of $138 \pm 54 \mathrm{~nm}$ (empty) and a slight increase in size value up to $156 \pm 82 \mathrm{~nm}$ after HRP encapsulation (Figure 2 ). These values were obtained before the purification of NERs

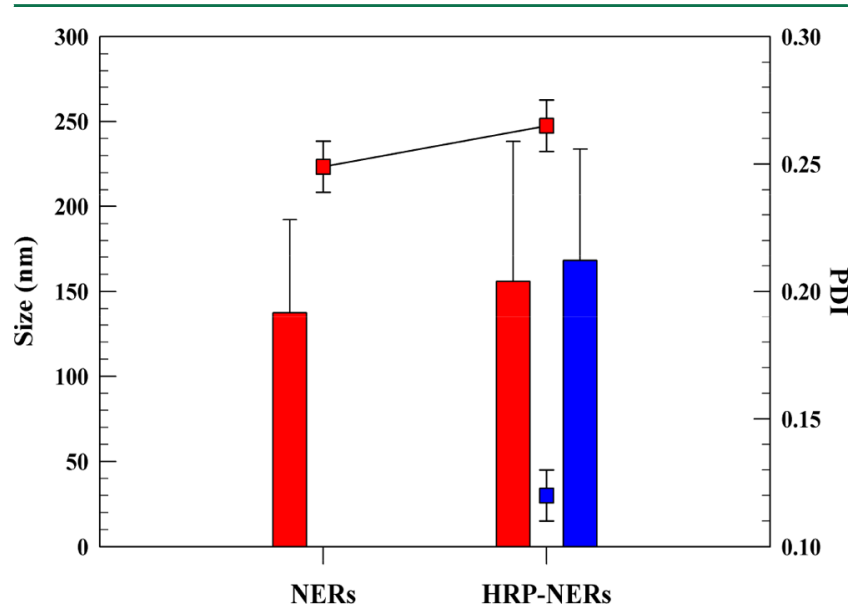

Figure 2. Dynamic light scattering analysis of size and polydispersity index (PDI) of empty nanoerythrosomes (NERs) and horseradish peroxidase loaded-nanoerythrosomes (HRP-NERs) before (red) and after (blue) purification through bag dialysis. Empty NERs were not purified before the analysis. The dialysis was carried out only for the HRP-NERs to remove the untrapped enzyme. The PDI of HRP-NERs decreased below 0.1 due to the removing of enzyme which is adsorbed on the surface of NERs and is not loaded from the nanovesicles. The resulting PDI shows a narrow sized distribution of HRP-NERs. The analysis was carried out using a Zetasizer Nano ZS (Malvern, UK) and represents the average \pm standard deviation (S.D.) from at least three independent measurements.

made up by direct extrusion. Conversely, HRP-NERs had an average size of $168 \pm 66 \mathrm{~nm}$ after purification (Figure 2). The small increase in size after the loading of HRP could depend on the high molecular weight of enzyme itself, ${ }^{35}$ which was confined in the outer compartment of nanoparticles. In addition, PDI values changed from $0.249 \pm 0.01$ of empty NERs to $0.265 \pm 0.01$ and $0.120 \pm 0.01$ of HRP-NERs before and after purification, respectively (Figure 2). The increase of PDI value after the loading of HRP could depend on the rearrangement of enzyme inside the nanoparticles. In fact, HPR was not completely dispersed inside the aqueous core of NERs but could partially adsorbed on the external bilayer. This distribution of enzyme in the colloidal nanoparticles was evident for unpurified NERs; therefore, the PDI decreased and was below 0.15 after purification due to the successful removing of the unentrapped HRP, which allowed to make narrow size distributed nanoparticles (Figure 2).

Dynamic light scattering (DLS) analysis was further endorsed by nanoparticle tracking analysis (NTA). NTA data of empty NERs were in agreement with the DLS results and showed a mean particle size of $154 \pm 1 \mathrm{~nm}$ for empty nanoparticles (Figure 3).

The mode and standard deviation of measurements were 144 \pm 8 and $43 \pm 1 \mathrm{~nm}$, respectively (Figure 3 and Table S2). Results demonstrated that NERs were fractionated in three major particle distributions with different diameter values as below reported: (i) $10 \%$ of the particles showed a mean diameter of $72 \pm 1 \mathrm{~nm}$; (ii) $50 \%$ had a mean diameter of $119 \pm$ $3 \mathrm{~nm}$; and (iii) $90 \%$ had a mean diameter of $181 \pm 2 \mathrm{~nm}$ (Figure 3 and Table S2). The three major distributions of

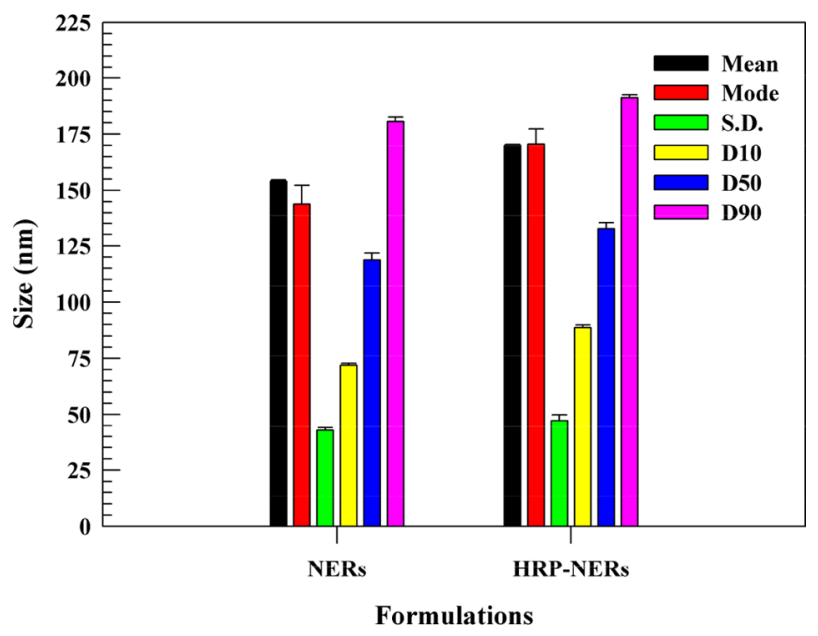

Figure 3. Nanoparticle tracking analysis (NTA) of (A) empty nanoerythrosomes or NERs and (B) horseradish peroxidase-loaded nanoerythrosomes or HRP-NERs: D10, D50, and D90 represent the average diameter of the 10,50 , and $90 \%$ of the nanoparticles. The analysis represents the average \pm standard deviation (S.D.) from at least three independent measurements.

nanoparticles depended on the origin of NERs. In fact, since these natural colloidal nanoparticles take origin from RBCs, it is not easy to control their size distribution accurately as with the synthetic nanoparticles. A slight increase of NTA analysis was carried out after loading of HRP inside NERs (Figure 3). The mode and standard deviation of measurements for the HPRNERs were $171 \pm 7$ and $47 \pm 3 \mathrm{~nm}$, respectively (Figure 3 and Table S2). HPR-NERs were also fractionated in three major particle distributions with different diameter values as below reported: (i) $10 \%$ of the particles showed a mean diameter of $89 \pm 1 \mathrm{~nm}$; (ii) $50 \%$ had a mean diameter of $133 \pm 3 \mathrm{~nm}$; and (iii) $90 \%$ had a mean diameter of $191 \pm 1 \mathrm{~nm}$ (Figure 3 and Table S2). Results were in agreement with DLS analysis (Figure 2) and demonstrated that the loading of enzyme provided a slight increase of average sizes and size distribution without affecting significantly the physicochemical parameters of NERs. The NTA analysis also calculated the final concentration of NERs as a function of average sizes in the sample suspension. ${ }^{34,35}$ Empty NERs showed a particle size concentration of $1.23 \times 10^{9} \mathrm{NERs} / \mathrm{mL}$ (Figure 3), whereas HPR-NERs had a particle size concentration of $1.38 \times 10^{9}$ NERs/ml (Table S2). Both for the DLS and NTA analysis were not necessary to dilute samples in order to avoid multiscattering phenomena because their concentrations were already at the magnitude of $\sim 1 \times 10^{9}$ particles $/ \mathrm{mL}$.

TEM images proved the limited thickness of the cancer cell derived vesicles $(\approx 4 \mathrm{~nm}){ }^{13}$ formed by direct extrusion, compared to NERs, and also their broad size distribution (Figure 1A, B).

TEM images showed that NERs loaded with the enzyme (Figure 4) were well distributed along the grid, presented a narrow size distribution, and a thick membrane $(\approx 11 \mathrm{~nm}),{ }^{17}$ suitable for drug delivery applications (Figure 4). These data were in agreement with empty NERs (Figure 1C-F). TEM analysis further demonstrated that the loading of HPR inside NERs did not modify the shape, size and size distribution of nanoparticles before (Figure 4A, C) and after (Figure 4B, D) their purification. Furthermore, NERs loaded with the enzyme 


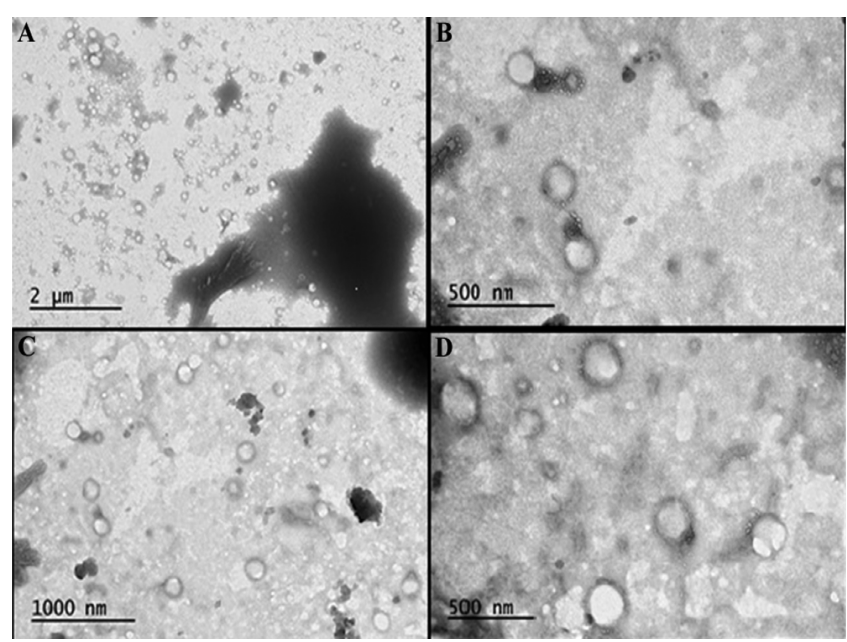

Figure 4. Transmission electron microscopy (TEM) images of horseradish peroxidase loaded-nanoerythrosomes (HRP-NERs). HRP-NERs (A, B) before and (C, D) after dialysis purification do not modify significantly their size and shape compared to the empty vesicles (see Figure 1). The TEM images are representative of three different and independent analyses.

preserved the characteristic biconcave discoidal shape of RBCs as herein reported for empty NERs (Figure 1F).

We further investigated the zeta potential of NERs before and after purification of colloidal nanoparticles. The zeta potential takes origin from interactions of the electric double layer surrounding the particle. ${ }^{36}$ The magnitude of the zeta potential gives an indication of the potential stability of the colloidal system. If all the particles in suspension have a large negative or positive zeta potential then they will tend to repel each other and there will be no tendency for the particles to aggregate. ${ }^{36}$ However, if the particles have low zeta potential values, then there will be no force to prevent the particles coming together and flocculating. ${ }^{36}$ The limit between stable and unstable suspensions is generally taken at either +30 or $-30 \mathrm{mV}$. Particles with zeta potential more positive than +30 $\mathrm{mV}$ or more negative than $-30 \mathrm{mV}$ are generally considered stable. The zeta potential values agreed data previously reported by other different groups. ${ }^{17,37} \mathrm{RBCs}$ have natural source, samples produced by sonication and extrusion methods in PBS or Milli-Q water ( $\mathrm{pH}$ 7.4) showed a very reproducible zeta potential value of ca. -20 and $-30 \mathrm{mV}$ (Table 1). These data were in agreement with those previously reported. ${ }^{37} \mathrm{ZP}$ values did not depend on the preparation methods and loading of HRP and are only affected by the composition of external membrane vesicles (Table 1). In fact, nanoparticles, made from cancer cell membranes, are less negative $(-20 \mathrm{mV})$ than NERs $(-30 \mathrm{mV})$ and these values are not affected by the purification and entrapment of HRP (Table 1). The average sizes, PDI (Figure S1A), and ZP (Figure S1B) of NERs and HPR-NERs are stable up to 3 days and increased from day 3 to 7 (Figure S1). Conversely, NERs and HPR-NERs are not stable in MilliQ-water (Figure S2), RPMI-1640 medium (Figure S3) and RPMI-1640 supplemented with FBS (Figure S4). The average sizes and PDI of NERs and HPR-NERs in Milli-Q-water ( Figure S2A), RPMI-1640 medium (Figure S3A), and RPMI1640 supplemented with FBS (Figure S4A), as well as their ZP values (Figures S2B, S3B, and S4B, respectively), increased at different time of incubations (days 0-7) compared to the native NERs and HPR-NERs (Figure S1). Differences in the average sizes, PDI, and ZP of NERs and HPR-NERs incubated in the aqueous medium and cell culture medium with or without FBS depended on the ionic strength and composition of medium, which may have favored the ionic interaction between RBC components of NERs, thus promoting the aggregation of nanoparticles. Interestingly, the presence of FBS in the cell culture medium may favor the self-assembling of serum components in nanoaggregates (Peak1, average sizes) of $\sim 30 \mathrm{~nm}$ (Figure S4A), which increased in sizes from day 3 to 7 of incubation times, while remaining stable (Peak2, average sizes) in HRP-NERs (Figure S4A).

Loading Efficiency of Nanocarriers. The loading of payloads is another important physicochemical parameter to be investigated because this value is strictly related to the therapeutic activity of nanoparticles. ${ }^{38}$ This is true for drugs, particularly anticancer ${ }^{39}$ and anti-inflammatory agents, ${ }^{40}$ peptides, ${ }^{41}$ and nucleic acid. ${ }^{38,42}$ Regarding the enzyme activity, although high encapsulation efficiencies are often desired, when enzymes preserve their catalytic activity, the maximum number of entrapped enzymes is not so important. ${ }^{18}$

The loading percentage of HRP inside NERs was calculated with an external calibration curve (Figure S5 and eq S3) using sequences of different standard solutions of HRP in sodium phosphate buffer $(25 \mathrm{mM}, \mathrm{pH} 7.4)$ (Table S1). The resulting calculated encapsulation efficiency \% (EE\%) of HRP is $58.2 \pm$ $2.91 \%$ (eq S2). This value demonstrates that NERs can entrap efficiently the HRP in their aqueous compartment.

Enzyme Assay and Kinetic of Nanocarriers. The catalytic activity of the NERs has been investigated using the Amplex Red (AR) according to standard protocols reported in other studies. ${ }^{13}$ In this method, the nonfluorescent AR has been oxidized by $\mathrm{H}_{2} \mathrm{O}_{2}$, in the presence of $\mathrm{HRP}$, producing a fluorescent end product, resorufin. ${ }^{13}$ In particular, the enzymatic reaction allows to follow the formation of the product because of its high fluorescence intensity as a function of time. This is clear evidence that the enzyme preserved its activity after entrapment into the NERs and after purification. Furthermore, the reaction kinetics can be monitored because the cell membrane itself is semipermeable and allowed the flow of molecules and substances on the inside and on the outside of the systems (Figures S6 and S7). ${ }^{13,43}$

Apparent kinetic constants $\left(K_{\mathrm{m}}\right.$ and $\left.V_{\max }\right)$ were obtained by fitting the Michaelis-Menten equation (eq S1) to plot the initial reaction rates versus the substrate concentrations using Origin version 8.6 Software (Table 2).

Experimental results highlight demonstrated a 2-fold increase of the $V_{\max }$ for the nanoreactor $\left(9141.66 \pm 189.35 \times 10^{-6} \mathrm{M}\right.$ $\mathrm{min}^{-1}$ ) compared to pure HRP in solution $(5740.81 \pm 37.67 \times$

Table 2. Enzyme Kinetics Parameters, Michaelis-Menten Constant and Maximum Reaction Rate Values of Pure Horseradish Peroxidase and Horseradish Peroxidase-Loaded Nanoerythrosomes (nanoreactor) Using Hydrogen Peroxide $\left(\mathrm{H}_{2} \mathrm{O}_{2}\right)$ and Amplex Red (AR) as Substrates ${ }^{a}$

$\begin{array}{lcc}\text { formulations } & V_{\max }\left(\times 10^{-6} \mathrm{M} \mathrm{min}^{-1}\right)^{b} & K_{\mathrm{m}}\left(\times 10^{-6} \mathrm{M}\right)^{c} \\ \text { HRP }^{d} & 5740.81 \pm 37.67 & 11.85 \pm 0.04 \\ \text { HPR-NERs }^{e} & 9141.66 \pm 189.35 & 9.50 \pm 0.22\end{array}$

${ }^{a}$ Results are the mean \pm standard deviation from at least three independent measurements. ${ }^{b} V_{\max }$, maximum reaction rate. ${ }^{c} K_{\mathrm{m}}$, Michaelis-Menten constant. ${ }^{d}$ HRP, pure horseradish peroxidase; ${ }^{e}$ HRP-NERs, horseradish peroxidase-loaded nanoerythrosomes. 


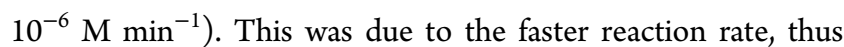
suggesting a high catalytic activity of the confined HRP within the membrane-enclosed compartment (Figure S7).

$K_{\mathrm{m}}$ low values were obtained for the nanoreactor $(9.50 \pm$ $\left.0.22 \times 10^{-6} \mathrm{M}\right)$ compared to the pure enzyme $(11.85 \pm 0.04 \times$ $\left.10^{-6} \mathrm{M}\right)$; but the improvements were not so pronounced like with $V_{\max }$ (Figure S6). This value is probably indicating not complete purification so the enzymatic reaction is affected also by free enzyme on the outside of the vesicles (Figure S6). Furthermore, it may not be possible that the confinement of HRP destroyed the integrity of the HRP, otherwise a low $V_{\max }$ value would have also been expected, and moreover, resorufin would not have been even formed and then would not have been possible to observe any variations of fluorescence ( Figure S7). In addition, it has already been investigated the possibility that the electrostatic interactions of enzyme and biocompatible materials, could provide the protein unfolding, which could decrease the enzyme catalytic activity. ${ }^{13,44}$ Because all the components of the nanoreactor (e.g., red blood cell membrane and HRP at $\mathrm{pH}$ 7.4) have negative charges (Table 1), as previously reported, ${ }^{37}$ thus this possibility can be excluded. ${ }^{13}$

Therefore, these results indicated the high substrate affinity to HRP inside NERs, due to the favorable interactions of HRP, $\mathrm{H}_{2} \mathrm{O}_{2}$, and $\mathrm{AR}$ within the cell membrane, as a result of confinement effects (Figure S7).

The kinetic data demonstrated that $V_{\max }$ and $K_{\mathrm{m}}$ of the conversion of HRP into fluorescent resorufin in the presence of Amplex Red were increased using the NERs Figure S7) compared to pure enzyme (Figure S6) in solution due the confinement of enzymatic reaction inside the aqueous compartment of NERs. This effect increased the interaction between enzyme and substrate and potentiates the reaction rate, thus providing a 2 -fold increase in enzyme efficiency compared to bulk solution ( Figure S7).

These results were in agreement with data previously reported, ${ }^{13}$ where undecylenic acid modified thermally hydrocarbonized silica nanoparticles (UnPSi) with or without coating with the cancer cell membranes allowed the access of AR and $\mathrm{H}_{2} \mathrm{O}_{2}$ inside the core of nanoparticles, their confinement and enzymatic reaction with HRP loaded in the aqueous core due to permeation of membranes forming the relative coating. ${ }^{45}$ In particular, UnPSi nanoparticles and nanoreactors decreased of 2- and 13-fold their $K_{\mathrm{m}}$ values compared to free enzyme due to the confinement of $\mathrm{H}_{2} \mathrm{O}_{2}$ inside the nanoreactors and the resulting high affinity of this substrate to HRP. ${ }^{13}$ Furthermore, pores of UnPSi nanoparticles loading HRP promoted the interaction between HRP and $\mathrm{H}_{2} \mathrm{O}_{2}$, and facilitated the collisions of $\mathrm{H}_{2} \mathrm{O}_{2}$ with pores, interface of particle surface, and cell membranes, thus resulting in a confinement process. ${ }^{46}$ These results were further reported in previous study demonstrating the higher affinity of substrates for enzymes after its immobilizing in polymeric nanoreactorrs. ${ }^{46}$ Conversely, the previous study demonstrated that the $V_{\max }$ increased $\sim 2$ fold and $\sim 3$ fold for UnPSi nanoparticles and nanoreactors, respectively, compared to free enzyme, thus suggesting high catalytic activity of HRP that is confined within the membrane compartment of UnPSi nanoparticles and nanoreactors. ${ }^{13}$ These data are in agreement with $V_{\max }$ of HRP-NERs (Table 2 ). The resulting increase of catalytic reaction depended on the high permeability of the membranes coating the UnPSi nanoparticles or forming nanoreactors, ${ }^{13}$ as in the case of NERs. In fact, previous data demonstrated that biological membranes were high permeable to $\mathrm{H}_{2} \mathrm{O}_{2}$, used for other purposes, ${ }^{47}$ and this high permeation enhanced the flow of $\mathrm{H}_{2} \mathrm{O}_{2}$ through the membrane and accelerated its local concentration as well as affinity to HRP, thus increasing the enzymatic catalysis. Conversely, low values of $V_{\max }$ obtained for porous silica particles and polymersomes, compare to free enzyme in solution, showed that a slow enzymatic reaction is carried out. $^{47-49}$ This decrease of catalytic reaction and the resulting slow reaction that occurred particularly in porous silica nanoparticles, as previously reported, ${ }^{13}$ depended on different factors such as modification of the enzyme structure, adsorption of the enzyme on the porous materials crowding effects and diffusion of molecules through the membranes. ${ }^{50,51}$ Furthermore, the decrease of $V_{\max }$ values for $\mathrm{H}_{2} \mathrm{O}_{2}$ depended on the disruption of the enzyme that was affected by the confinement of HRP inside the nanoreactor compartment, ${ }^{13}$ or due to the diffusional resistance of substrates. ${ }^{52-54}$ In such a way, we previously demonstrated that UnPSi nanoparticles coated with cancer cell membranes favored the interaction between $\mathrm{AR}$ with the hydrophobic counterpart of bilayer membrane and hindered the enzymatic interaction with HRP, ${ }^{13}$ thus causing slow values of $V_{\max }$ of nanoparticles compared to the free HPR in solution. ${ }^{55}$

Conversely, spherical polyelectrolyte brushes nanoparticles of $200 \mathrm{~nm}$ in size can also affect the Michaelis-Menten kinetic constants allowed the immobilization of enzymes on the surface of colloidal nanopartilces and decreased their enzymatic activity, compared to the free enzyme in solution, due to the protein-protein interaction occurred using the nanreactors. ${ }^{56}$

Triblock copolymers of poly(2-methyloxazoline)-block-poly(dimethylsiloxane)-block-(2-methyloxazoline) forming PEGylated liposome based nanoreactors affected the Michaelis-Menten kinetic constants of various entrapped enzymes, i.e., inosine, adenosine, guanosine, thus potentiating the enzyme-substrate interaction and improving the membrane transport and activity as a function of porine to polymer ratio in the nanoreactor membrane. ${ }^{57}$

Results demonstrated that the red blood cell membrane allowed the substrates ( $\mathrm{AR}$ and $\mathrm{H}_{2} \mathrm{O}_{2}$ ) to access the encapsulated HRP, suggesting that the membrane was permeable and allowed the flow of molecules (Figure S7).

\section{CONCLUSIONS}

In conclusion, in this work cancer and red blood cells' membranes have been derived from their source material, i.e., breast cancer cells (MDA-MB-231) and red blood cells. Their physicochemical properties (e.g., average sizes, PDI, morphology) have been studied in order to exploit their potentiality as natural drug delivery systems and, more in detail, as nanoreactors (i.e., miniaturized reaction vessels). ${ }^{13}$ The source material has been selected to take advantages of the homotypic binding of cancer cells and the inherent biocompatibility, long systemic circulation and stealth capability specific of RBCs. The physicochemical characterization showed that both the natural colloidal nanoparticles, synthesized and physicochemical characterized by different methods, present a size smaller than $200 \mathrm{~nm}$ and a narrow size distribution with a PDI value smaller than 0.3. Moreover, TEM studies demonstrated that NERs had very thick membrane $(\approx 11 \mathrm{~nm})$, appropriate to deliver a wide variety of therapeutic agents and preserved the characteristic biconcave disc shape of red blood cells, whereas cancer cell derived vesicles might be suitable only for the coating of nanoparticles. 
The loading of HRP in this thin and ultrasoft compartment of NERs could cause a fast catalytic reaction, therefore this study, as a proof of concept, could make nanoreactors an attractive field of study to have a better comprehension of biological processes and, eventually, to develop a new generation of biomimetic nanotherapeutic agents.

\section{ASSOCIATED CONTENT}

\section{S Supporting Information}

The Supporting Information is available free of charge on the ACS Publications website at DOI: 10.1021/acsbiomaterials.7b00944.

Detailed Materials and Methods section of different procedures that are used to perform the various experiments; experimental details and physicochemical characterization results of cancer cell membranes and resulting nanocarriers; experimental details and secondary physicochemical characterization results of enzyme assay and kinetic of nanoerythrosomes (PDF)

\section{AUTHOR INFORMATION}

\section{Corresponding Authors}

*E-mail: helder.santos@helsinki.

*E-mail: c.celia@unich.it.

ORCID $\odot$

Hélder A. Santos: 0000-0001-7850-6309

\section{Author Contributions}

${ }^{\dagger}$ V.B. and A.P. contributed equally. The manuscript was written through contributions of all authors. All authors have given approval to the final version of the manuscript.

\section{Notes}

The authors declare no competing financial interest.

\section{ACKNOWLEDGMENTS}

This manuscript was partially supported by FAR 2017, MIUR grant, University of Chieti-Pescara “G. d'Annunzio", Chieti, Italy. The financial support from the Tekes large strategic research opening 3i Regeneration (project no. 40395/13) is gratefully acknowledged. Prof. H. Zhang acknowledges financial support from the Academy of Finland (Decision 297580), Jane and Aatos Erkko Foundation (Grant 4704010) and Sigrid Jusélius Foundation (Decision 28001830K1). Prof. H. A. Santos acknowledges financial support from the University of Helsinki Research Funds, the HiLIFE Research Funds, the Sigrid Juselius Foundation (Grant 4704580), and the European Research Council under the European Union's Seventh Framework Programme (FP/2007-2013; Grant 310892).

\section{ABBREVIATIONS}

NERs, nanoerythrosomes; HRP, horseradish peroxidase; $V_{\max }$ maximal velocity; $K_{\mathrm{m}}$, Michaelis constant; MDA-MB-231, breast cancer cells; RBCs, red blood cells; PDI, polydispersity index; TLE, thin layer evaporation; TEM, transmission electron microscopy; DLS, dynamic light scattering; NTA, nanoparticle tracking analysis; AR, amplex red

\section{REFERENCES}

(1) Balasubramanian, V.; Herranz-Blanco, B.; Almeida, P. V.; Hirvonen, J.; Santos, H. A. Multifaceted polymersome platforms: spanning from self-assembly to drug delivery and protocells. Prog. Polym. Sci. 2016, 60, 51-85.
(2) Luisi, P. L. The Emergence of Life: From Chemical Origins to Synthetic Biology; Cambridge University Press: Cambridge, UK; 2006; p 332.

(3) Mann, S. Systems of creations: the emergence of life from nonliving matter. Acc. Chem. Res. 2012, 45 (12), 2131-2141.

(4) Stano, P. Synthetic biology of minimal living cell: primitive cell models and semi-synthetic cells. Syst. Synth. Biol. 2010, 4 (3), 149-56.

(5) Walde, P.; Wick, R.; Fresta, M.; Mangone, A.; Luisi, P. L. Autopoietic self-reproduction of fatty acid vesicles. J. Am. Chem. Soc. 1994, 116 (26), 11649-11654.

(6) Dzieciol, A. J.; Mann, S. Designs for life: protocell models in the laboratory. Chem. Soc. Rev. 2012, 41 (1), 79-85.

(7) Ichihashi, N.; Yomo, T. Positive roles of compartmentalization in internal reactions. Curr. Opin. Chem. Biol. 2014, 22, 12-17.

(8) Gao, W.; Zhang, L. Coating nanoparticles with cell membranes for targeted drug delivery. J. Drug Target. 2015, 23 (7-8), 619-626.

(9) Luk, B. T.; Zhang, L. Cell membrane camouflaged nanoparticles for drug delivery. J. Controlled Release 2015, 220, 600-607.

(10) Hu, C. M.; Fang, R. H.; Zhang, L. Erythrocyte-inspired delivery systems. Adv. Healthcare Mater. 2012, 1 (5), 537-547.

(11) Hu, C. M.; Zhang, L.; Aryal, S.; Cheung, C.; Fang, R. H.; Zhang, L. Erythrocyte membrane-camouflaged polymeric nanoparticle as a biomimetic delivery platform. Proc. Natl. Acad. Sci. U. S. A. 2011, 108 (27), 10980-10985.

(12) Zhang, H. Erythrocytes in nanomedicine: an optimal blend of natural and synthetic materials. Biomater. Sci. 2016, 4 (7), 1024-1031.

(13) Balasubramanian, V.; Correia, A.; Zhang, H.; Fontana, F.; Mäkilä, E.; Salonen, J.; Hirvonen, J.; Santos, H. A. Biomimetic engineering using cancer cell membranes for designing compartmentalized nanoreactors with organelle-like functions. Adv. Mater. 2017, 29 (11), 1605375.

(14) Biagiotti, S.; Paoletti, M. F.; Fraternale, A.; Rossi, L.; Magnani, M. Drug Delivery by Red Blood Cells. IUBMB Life 2011, 63 (8), 621631.

(15) Godfrin, Y.; Bax, B. E. Enzyme bioreactors as drugs. Drugs Future 2012, 37 (4), 263-272.

(16) Mohandas, N.; Gallagher, P. G. Red cell membrane: past, present and future. Blood 2008, 112 (10), 3939-3948.

(17) Deák, R.; Mihály, J.; Szigyártó, I. C.; Wacha, A.; Lelkes, G.; Bóta, A. Physicochemical characterization of artificial nanoerythrosomes derived from erythrocyte ghost membranes. Colloids Surf., B 2015, 135, $225-234$.

(18) Tanner, P.; Egli, S.; Balasubramanian, V.; Onaca, O.; Palivan, C. G.; Meier, W. Can polymeric vesicles that confine enzymatic reactions act as simplified organelles? FEBS Lett. 2011, 585 (11), 1699-1706.

(19) Adumeau, L.; Genevois, C.; Roudier, L.; Schatz, C.; Couillaud, F.; Mornet, S. Impact of surface grafting density of PEG macromolecules on dually fluorescent silica nanoparticles used for the in vivo imaging of subcutaneous tumors. Biochim. Biophys. Acta, Gen. Subj. 2017, 1861 (6), 1587-1596.

(20) Campbell, R. B.; Fukumura, D.; Brown, E. B.; Mazzola, L. M.; Izumi, Y.; Jain, R. K.; Torchilin, V. P.; Munn, L. L. Cationic charge determines the distribution of liposomes between the vascular and extravascular compartments of tumors. Cancer Res. 2002, 62 (23), 6831-6836.

(21) Decuzzi, P.; Godin, B.; Tanaka, T.; Lee, S. Y.; Chiappini, C.; Liu, X.; Ferrari, M. Size and shape effects in the biodistribution of intravascularly injected particles. J. Controlled Release 2010, 141 (3), $320-327$.

(22) Takeuchi, I.; Nobata, S.; Oiri, N.; Tomoda, K.; Makino, K. Biodistribution and excretion of colloidal gold nanoparticles after intravenous injection: effects of particle size. Bio-Med. Mater. Eng. 2017, 28 (3), 315-323.

(23) Herranz-Blanco, B.; Shahbazi, M. A.; Correia, A. R.; Balasubramanian, V.; Kohout, T.; Hirvonen, J.; Santos, H. A. pHswitch nanoprecipitation of polymeric nanoparticles for multimodal cancer targeting and intracellular triggered delivery of doxorubicin. Adv. Healthcare Mater. 2016, 5 (15), 1904-1916. 
(24) Paolino, D.; Cosco, D.; Racanicchi, L.; Trapasso, E.; Celia, C.; Iannone, M.; Puxeddu, E.; Costante, G.; Filetti, S.; Russo, D.; Fresta, M. Gemcitabine-loaded PEGylated unilamellar liposomes vs GEMZAR: biodistribution, pharmacokinetic features and in vivo antitumor activity. J. Controlled Release 2010, 144 (2), 144-150.

(25) Pasut, G.; Paolino, D.; Celia, C.; Mero, A.; Joseph, A. S.; Wolfram, J.; Cosco, D.; Schiavon, O.; Shen, H.; Fresta, M. Polyethylene glycol (PEG)-dendron phospholipids as innovative constructs for the preparation of super stealth liposomes for anticancer therapy. J. Controlled Release 2015, 199, 106-113.

(26) Figueiredo, P.; Ferro, C.; Kemell, M.; Liu, Z.; Kiriazis, A.; Lintinen, K.; Florindo, H. F.; Yli-Kauhaluoma, J.; Hirvonen, J.; Kostiainen, M. A.; Santos, H. A. Functionalization of carboxylated lignin nanoparticles for targeted and $\mathrm{pH}$-responsive delivery of anticancer drugs. Nanomedicine (London, U. K.) 2017, 12 (21), 2581-2596.

(27) Paolino, D.; Cosco, D.; Gaspari, M.; Celano, M.; Wolfram, J.; Voce, P.; Puxeddu, E.; Filetti, S.; Celia, C.; Ferrari, M.; Russo, D.; Fresta, M. Targeting the thyroid gland with thyroid-stimulating hormone (TSH)-nanoliposomes. Biomaterials 2014, 35 (25), 71017109 .

(28) Reuter, L. J.; Shahbazi, M. A.; Mäkilä, E. M.; Salonen, J. J.; Saberianfar, R.; Menassa, R.; Santos, H. A.; Joensuu, J. J.; Ritala, A. Coating nanoparticles with plant-produced transferrin-hydrophobin fusion protein enhances their uptake in cancer cells. Bioconjugate Chem. 2017, 28 (6), 1639-1648.

(29) Villa, C. H.; Anselmo, A. C.; Mitragotri, S.; Muzykantov, V. Red blood cells: supercarriers for drugs, biologicals, and nanoparticles and inspiration for advanced delivery systems. Adv. Drug Delivery Rev. 2016, 106, 88-103.

(30) Verma, D. D.; Verma, S.; Blume, G.; Fahr, A. Particle size of liposomes influences dermal delivery of substances into skin. Int. J. Pharm. 2003, 258, 141-151.

(31) Calvagno, M. G.; Celia, C.; Paolino, D.; Cosco, D.; Iannone, M.; Castelli, F.; Doldo, P.; Fresta, M. Effects of lipid composition and preparation conditions on physical-chemical properties, technological parameters and in vitro biological activity of gemcitabine-loaded liposomes. Curr. Drug Delivery 2007, 4 (1), 89-101.

(32) Zhang, X.; Jing, L.; Chang, F.; Chen, S.; Yang, H.; Yang, Q. Positional immobilization of $\mathrm{Pd}$ nanoparticles and enzymes in hierarchical yolk-shell@shell nanoreactors for tandem catalysis. Chem. Commun. (Cambridge, U. K.) 2017, 53 (55), 7780-7783.

(33) Debnath, A.; Thakkar, F. M.; Maiti, P. K.; Kumaran, V.; Ayappa, K. G. Laterally structured ripple and square phases with one and two dimensional thickness modulations in a model bilayer system. Soft Matter 2014, 10 (38), 7630-7607.

(34) Jackson, M. B. Minimum membrane bending energies of fusion pores. J. Membr. Biol. 2009, 231 (2-3), 101-115.

(35) Veitch, N. C. Horseradish peroxidase: a modern view of a classic enzyme. Phytochemistry 2004, 65 (3), 249-259.

(36) Hole, P.; Sillence, K.; Hannell, C.; Maguire, C. M.; Roesslein, M.; Suarez, G.; Capracotta, S.; Magdolenova, Z.; Horev-Azaria, L.; Dybowska, A.; Cooke, L.; Haase, A.; Contal, S.; Manø, S.; Vennemann, A.; Sauvain, J. J.; Staunton, K. C.; Anguissola, S.; Luch, A.; Dusinska, M.; Korenstein, R.; Gutleb, A. C.; Wiemann, M.; Prina-Mello, A.; Riediker, M.; Wick, P. Interlaboratory comparison of size measurements on nanoparticles using nanoparticle tracking analysis (NTA). J. Nanopart. Res. 2013, 15, 2101.

(37) Kestens, V.; Bozatzidis, V.; De Temmerman, P. J.; Ramaye, Y.; Roebben, G. Validation of particle tracking analysis method for the size determination of nano- and microparticles. J. Nanopart. Res. 2017, 19 (8), 271.

(38) Hiemenz, P. C.; Rajagopalan, R. Principles of Colloid and Surface Chemistry, 3rd ed.; Marcel Dekker: New York, 1997.

(39) Kuo, Y. C.; Wu, H. C.; Hoang, D.; Bentley, W. E.; D’Souza, W. D.; Raghavan, S. R. Colloidal properties of nanoerythrosomes derived from bovine red blood cells. Langmuir 2016, 32 (1), 171-179.

(40) Kumari, A.; Singla, R.; Guliani, A.; Yadav, S. K. Nanoencapsulation for drug delivery. EXCLI J. 2014, 13, 265-286.
(41) Kale, M.; Suruse, P.; Singh, R.; Malhotra, G.; Raut, P. Effect of size reduction techniques on doxorubicin hydrochloride loaded liposomes. Int. J. Biol. Pharm. Res. 2012, 3, 308-316.

(42) Asthana, A.; Chauhan, A. S.; Diwan, P. V.; Jain, N. K. Poly (amidoamine) (PAMAM) dendritic nanostructures for controlled sitespecific delivery of acidic anti-inflammatory active ingredient. AAPS PharmSciTech 2005, 6, E536-E542.

(43) Felnerova, D.; Viret, J. F.; Gluck, R.; Moser, C. Liposomes and virosomes as delivery systems for antigens, nucleic acids and drugs. Curr. Opin. Biotechnol. 2004, 15, 518-529.

(44) Funhoff, A. M.; Monge, S.; Teeuwen, R.; Koning, G. A.; Schuurmans-Nieuwenbroek, N. M.; Crommelin, D. J.; Haddleton, D. M.; Hennink, W. E.; van Nostrum, C. F. PEG shielded polymeric double-layered micelles for gene delivery. J. Controlled Release 2005, 102, 711-724.

(45) Piwonski, H. M.; Goomanovsky, M.; Bensimon, D.; Horovitz, A.; Haran, G. Allosteric inhibition of individual enzyme molecules trapped in lipid vesicles. Proc. Natl. Acad. Sci. U. S. A. 2012, 109 (22), E1437-E1443.

(46) Chen, Q.; Schönherr, H.; Vancso, G. J. Block-copolymer vesicles as nanoreactors for enzymatic reactions. Small 2009, 5 (12), 14361445.

(47) Bienert, G. P.; Schjoerring, J. K.; Jahn, T. P. Membrane transport of hydrogen peroxide. Biochim. Biophys. Acta, Biomembr. 2006, 1758 (8), 994-1003.

(48) Cao, X.; Li, Y.; Zhang, Z.; Yu, J.; Qian, J.; Liu, S. Catalytic activity and stability of glucose oxidase/horseradish peroxidase coconfined in macroporous silica foam. Analyst 2012, 137 (24), 57855791.

(49) Orosco, M. M.; Pacholski, C.; Sailor, M. J. Real-time monitoring of enzyme activity in a mesoporous silicon double layer. Nat. Nanotechnol. 2009, 4 (4), 255-258.

(50) Lee, C. H.; Lin, T. S.; Mou, C. Y. Mesoporous materials for encapsulating enzymes. Nano Today 2009, 4, 165-179.

(51) Liu, J.; Yang, Q.; Li, C. Towards efficient chemical synthesis via engineering enzyme catalysis in biomimetic nanoreactors. Chem. Commun. (Cambridge, U. K.) 2015, 51 (72), 13731-13739.

(52) Tanner, P.; Onaca, O.; Balasubramanian, V.; Meier, W.; Palivan, C. G. Enzymatic cascade reactions inside polymeric nanocontainers: a means to combat oxidative stress. Chem. - Eur. J. 2011, 17 (16), 45524560.

(53) Gupta, N.; Shrivastava, A.; Sharma, R. K. Silica nanoparticles coencapsulating gadolinium oxide and horseradish peroxidase for imaging and therapeutic applications. Int. J. Nanomed. 2012, 7, 5491500 .

(54) Patterson, D. P.; Prevelige, P. E.; Douglas, T. Nanoreactors by programmed enzyme encapsulation inside the capsid of the bacteriophage P22. ACS Nano 2012, 6 (6), 5000-5009.

(55) Zhao, B.; Summers, F. A.; Mason, R. P. Photooxidation of Amplex Red to resorufin: implications of exposing the Amplex Red assay to light. Free Radical Biol. Med. 2012, 53 (5), 1080-1087.

(56) Neumann, T.; Haupt, B.; Ballauff, M. High activity of enzymes immobilized in colloidal nanoreactors. Macromol. Biosci. 2004, 4, 1316.

(57) Ranquin, A.; Versées, W.; Meier, W.; Steyaert, J.; Van Gelder, P. Therapeutic nanoreactors: combining chemistry and biology in a novel triblock copolymer drug delivery system. Nano Lett. 2005, 5 (11), $2220-2224$ 